\title{
A Southern Digital Sky Survey Using the UK Schmidt Telescope
}

\author{
Bruce A. Peterson \\ Mt. Stromlo and Siding Spring Observatories, Institute for Advanced \\ Study, The Australian National University, Private Bag, Weston \\ A.C.T. 2011, Australia
}

\begin{abstract}
Two CCD detector systems for the U. K. Schmidt Telescope that would allow a southern digital sky sulvey of faint objects to be carried out are described.
\end{abstract}

\section{Introduction}

Existing photographic surveys will provide targets for a redshift survey, using the 400 fibre $2 \mathrm{dF}$ system on the Anglo-Australian Telescope, that will cover 100 $\times 100$ square degrees with $10^{5}$ galaxies down to $B \sim 19.5$ and $z \sim 0.1$. However, a fainter survey is required in order to provide targets for a sparsely sampled redshift survey of $10^{4}$ galaxies down to $z \sim 0.3$ that will be used to study the evolution of galaxy clustering.

The linearity and dynamic range of CCDs make it possible to obtain images of fainter objects than can be obtained with individual photographic plates. CCDs also have greater quantum efficiency than photographic plates, especially at longer wavelengths, allowing shorter exposures.

This paper describes two approaches to a southern digital sky survey using CCDs on the UK Schmidt Telescope that would produce the required targets for the $z \sim 0.3$ redshift survey.

\section{A Drift Scanning System}

Shectman (1994) has proposed that from one to three large format $(5000 \times 5000)$ $12 \mu \mathrm{m} /$ pixel CCDs be mounted in the focal plane of a large Schmidt telescope and used for a drift scan sky survey. This approach has the advantage that there is no time lost while the large CCDs are being read out, and that objects with low surface brightness are more easily detected.

Drift scanning is done with the telescope not tracking. As the light image is carried across the CCD by the Earth's rotation, the charge image is moved along a column and kept under the light image by clocking the CCD. When the two images reach the last row, the charge is shifted into the serial register and then read out. Thus the CCD is continuously read out while drift scanning and there is no dead time. Because the charge image is accumulated in every pixel in a column along the scan direction, there are no CCD induced variations in 
the scan direction. Flat fielding only requires a correction for column to column average sensitivity variations.

The full system would drift scan using three large CCDs, one positioned north and another south of the central CCD. A gap slightly smaller (to allow overlap) than the north-south extent of a single CCD would separate the north and south CCDs from the central one. After one scan was completed, the telescope would be offset in declination so as to position the CCDs in the gaps of the previous scan, and offset west in right ascension so as to scan the same range of right ascension again, filling in the gaps. With a $5 \mathrm{k} \times 5 \mathrm{k}$ CCD having $12 \mu \mathrm{m}$ square pixels, about 43 square degrees an hour could be scanned, with an effective exposure time of $\sim 5$ minutes. A single scan would last a few hours, not all night.

Using such large CCDs introduces several difficulties. As discussed by Zaritsky (1993) in his proposal for a drift scanning system for the Las Campanas 40 inch telescope, the CCD must rotate and track in declination. This is accomplished by rotating the CCD continuously during the integration (a few degrees) and providing a linear motion (a few $\mathrm{cm}$ ) in the declination direction. Shectman (1994) has pointed out that the field-flattener lens must not introduce distortion. A further difficulty is the high cost of such large CCDs.

\section{A Large Area Mosaic}

Connroy (1994) has looked at the feasibility of mounting a $3 \times 4$ mosaic of 2048 $\times 204815 \mu \mathrm{m} /$ pixel CCDs on a spherical cold surface under a spherical silicadomed shell, $8 \mathrm{~mm}$ thick. Such a configuration could be used in a drift scanning mode or be used to make mosaic images. The fragility of the thin shaped window which is required in order to maintain image quality is a concern.

Other focal plane configurations and thermoelectric cooling (Petrick 1987) are being considered. The CCDs for this approach are available and cold testing will begin in a few weeks.

\section{References}

Conroy P., 1994, Schmidt Telescope CCD Project, private communication

Petrick S. W., 1987, Optical Engineering, 26, 965

Shectman S., 1994, A Drift Scanning Survey Using a 48 inch Schmidt Telescope, private communication

Zaritsky D., 1993, The Tracking Camera and Low Surface Brightness Galaxies, private communication

\section{Discussion}

Malin: You seem to be offering a survey detector that attempts to cover 40 square degrees in an hour, using a detector with $12 \mu \mathrm{m}$ pixels. Would you not be able to achieve equivalent or better coverage, by using e.g. Tech Pan film which has a detector quantum efficiency of $\sim 10 \%, 5 \mu \mathrm{m}$ pixels and digitally 
stacking several exposures. $z=\mathbf{0 . 3}$ galaxies are right at the limit of the spatial resolution of the UKST, so $12 \mu \mathrm{m}$ pixles would not do the right job.

Peterson: By working with CCDs in I-band, we require about one fifth of the telescope time to produce a survey in digital form. By using films, you would have the additional problems of digitization and adding with proper weighting. The spatial resolution would be determined by the seeing and telescope optics in either case.

Collins: Can you comment on whether this survey will have adequate spatial resolution with pixel size $\sim 1$ arcsec?

Peterson: Experience with the AAT $\mathrm{f} / 1$ prime focus CCD camera indicates that we can do star-galaxy separations to $z \sim 0.3$, which is our target for this survey. 